\title{
IoT Driven Automated Object Detection Algorithm for Urban Surveillance System in Smart City
}

\author{
D.V.B Pragna, D.Laxma Reddy, SVS Prasad
}

\begin{abstract}
Automated object detection algorithm is an important research challenge in intelligent urban surveillance systems for Internet of Things (IoT) and smart cities applications. In particular, smart vehicle license plate recognition and vehicle detection are recognized as core research issues of these IoTdriven intelligent urban surveillance systems. They are key techniques in most of the traffic related IoT applications, such as road traffic real-time monitoring, security control of restricted areas, automatic parking access control, searching stolen vehicles, etc. In this paper, we propose a novel unified method of automated object detection for urban surveillance systems. We use this novel method to determine and pick out the highest energy frequency areas of the images from the digital camera imaging sensors, that is, either to pick the vehicle license plates or the vehicles out from the images. The other sensors like flame and ultrasonic sensor are used to monitor nearby objects. Our proposed method can not only help to detect object vehicles rapidly and accurately, but also can be used to reduce big data volume needed to be stored in urban surveillance systems
\end{abstract}

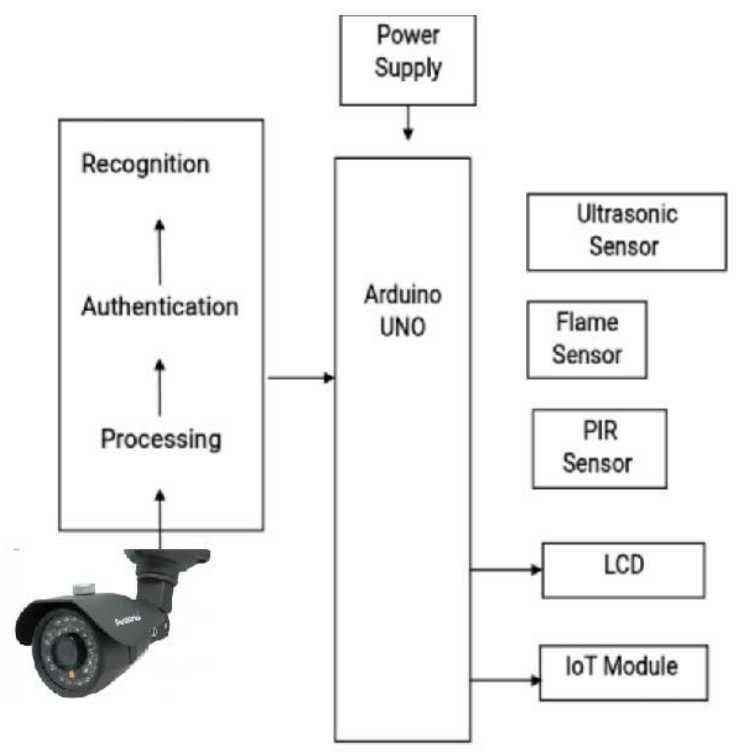

FIG - 2.1 Block Diagram

\section{I.BLOCK DIAGRAM AND WORKING}

Mechanized article location calculation is a significant research challenge in savvy urban observation frameworks for Internet of Things (IoT) and shrewd urban communities' applications. Specifically, keen vehicle tag acknowledgment and vehicle location are perceived as center research issues of these IoT-driven smart urban observation frameworks. They are key procedures in the majority of the traffic related IoT applications, for example, street traffic ongoing observing, security control of confined regions, programmed leaving access control, looking stolen vehicles, and so forth In this paper, we propose a novel brought together technique for mechanized item location for urban reconnaissance frameworks. We utilize this novel strategy to decide and choose the most elevated vitality recurrence zones of the pictures from the computerized camera imaging sensors, that is, either to pick the vehicle tags or the vehicles out from the pictures. Different sensors like fire and ultrasonic sensor are utilized to screen close-by articles. Our proposed strategy can not just recognize object vehicles quickly and precisely, yet in addition can be utilized to lessen huge information volume should have been put away in urban reconnaissance frameworks.

The flame sensor and ultrasonic sensor are connected to the Arduino controller. These sensors provide necessary information's like nearby vehicles etc. and the data are passed to the controller. The PIR sensor detects the motion on nearby surroundings and the data's are updated to the IoT. The controller enables the camera and used to detect the vehicle number and update the information to the IoT. Hence this surveillance system provides complete protection in the cities.

Revised Manuscript Received on 14 August, 2019.

D.V.B Pragna, PG Student, Department of ECE,MLR Institute of Technology, Hyderabad, Telangana, India.

D.Laxma Reddy, Associate professor, Department of ECE, MLR Institute of Technology, Hyderabad, Telangana, India.

SVS Prasad, Professor, Department of ECE, MLR Institute of Technology, Hyderabad, Telangana, India. 


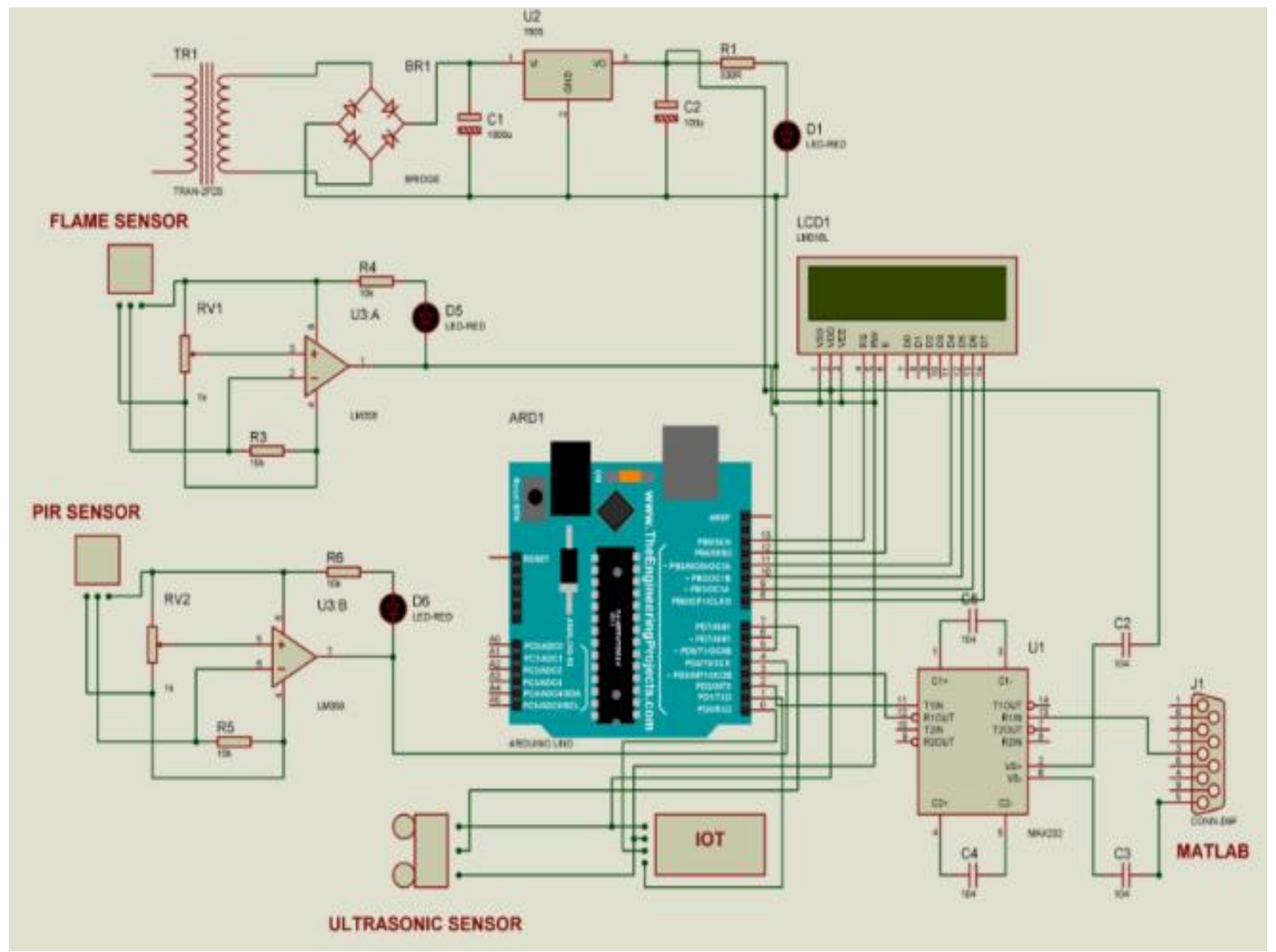

FIG -3.1 Image of Working drone

- Memory

Presently, letus see thesubtleties of thedifferent structure squaresof the equipment ofan implanted framework.As appeared inFig. thestructuresquares are;

- InputDevices

- Outputgadgets

- Communicationinterfaces· Application-specific circuitry

- CentralProcessing Unit
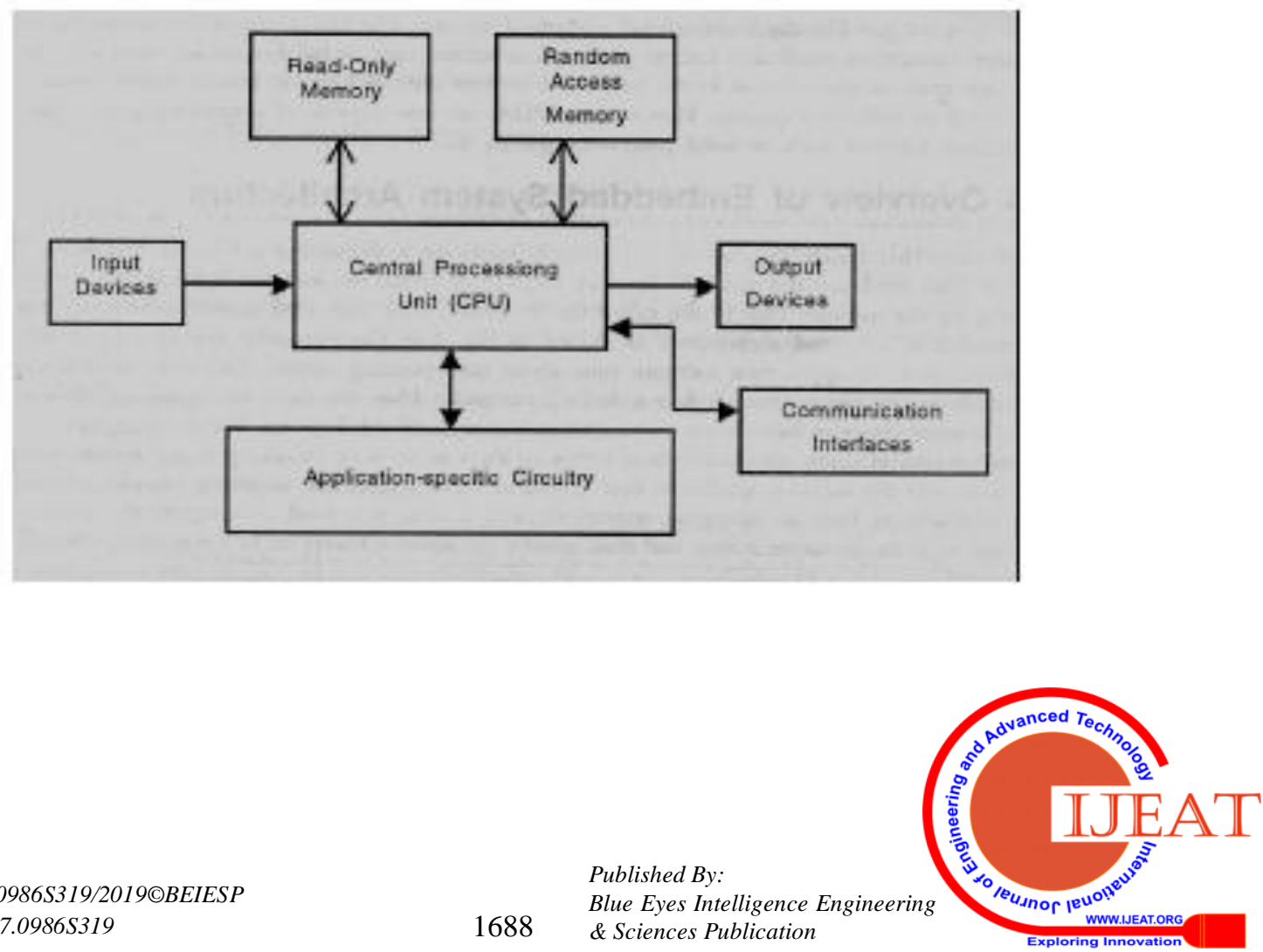


\section{Focal Processing Unit (CPU):}

The Central ProcessingUnit (processor, inshort) can beany of theaccompanying: microcontroller, chipor Digital SignalProcessor (DSP).

\section{Memory:}

Thememory is classifiedas Random AccessMemory (RAM) andRead Only Memory(ROM). The substanceof the RAM willbe deleted ifcontrol is changedoff to thechip, though ROMholds the substanceregardless of whetherthe power isturned off.

\section{Info Devices:}

Incontrast to the workareas, the infogadgets to aninstalled framework haveconstrained ability.Therewill be noconsole or amouse, and subsequentlycommunicating with theimplanted framework isno simple assignment.Many implanted frameworkswill have a littlekeypad-you press onekey to give a particularorder. A keypadmight be utilized to includejust the digits.

\section{Yield Devices:}

Theyield gadgets ofthe installed frameworksadditionally have extremely constrainedcapacity. Some installedframeworks will havea couple of LightEmitting Diodes (LEDs) todemonstrate the wellbeingstatus of theframework modules, orfor visual signof alerts. Alittle Liquid CrystalDisplay (LCD) maylikewise be utilizedto show somesignificant parameters.

\section{Correspondence Interfaces:}

Theinstalled frameworks mayneed to, collaboratewith other insertedframeworks at theymay need totransmit information toa work area.To encourage this,the inserted frameworksare furnished withone or acouple of correspondenceinterfaces, for example,RS232, RS422, RS485,Universal Serial Bus(USB), and IEEE1394, Ethernet andso forth.

ressively used responsible for model vehicles and flying machine. Presently, these $2.4 \mathrm{GHz}$ frameworks are being made by most radio makers. These radio frameworks go from a few thousand dollars, right down to under US\$30 for a few.Propellers are comparable in aerofoil segment to a low-drag wing and accordingly are poor in activity when at other than their ideal approach. Accordingly, a few propellers utilize a variable pitch instrument to modify the cutting edges' pitch edge as motor speed and air ship speed are changed.

\section{MODULES}

\subsection{POWERSUPPLY:}

Thepower supply area is thesegment which give $+5 \mathrm{~V}$ to the segments towork. IC LM7805is utilized forgiving a steady intensity of $+5 \mathrm{~V}$.

Air ship with traditional propellers, along these lines, don't generally fly quicker than Mach 0.6.

\subsection{MICROCONTROLLER:}

A Microcontroller isa PC on-a-chip used tocontrol electronic gadgets.It is a sortof microchip underscoringindependence and cost-adequacy, ratherthan a universallyuseful chip (the benevolentutilized in aPC). A run ofthe mill microcontroller containsall the memoryand interfaces requiredfor a straightforwardapplication, though auniversally useful microchiprequires extra chipsto give thesecapacities.

\subsection{Arduino UNO:}

Arduino/genuinoUnois a microcontrollerboard dependent ontheatmega328p(datasheet).It has 14advancedinfo/yield pins(of which6canbe utilizedaspwm yields),6 simple datasources, a $16 \mathrm{MHz}$ quartz preciousstone, a usbassociation, a powerjack, an icspheader and areset catch. Itcontains everything expectedto help themicrocontroller; basically interfaceit to aPC with ausb link orpower it withan air conditionerto-dc connector orbattery to begin..You can tinkerwith your unowithout agonizing alot over accomplishingsomething incorrectly, mostdire outcome imaginableyou can tradethe chip fora couple ofdollars and beginonce again oncemore.

\subsection{LIQUID CRYSTAL DISPLAY:}

LCD (LiquidCrystal Display) screenis an electronicshowcase module andlocate a widescope of uses.A $16 \times 2$ LCDshow is fundamentalmodule and isall around regularlyutilized in differentgadgets and circuits. These modules arefavored more thanseven portions andother multi sectionLEDs. The reasonsbeing: LCDs areefficient; effectively programmable;have no restrictionof showing uncommonand even customcharacters (not atall like inseven sections), activitys,etc.A 16x2LCD implies itcan show 16 characters for everyline and thereare 2 suchlines. In thisLCD each characterisshownin $5 \times 7$ pixelgrid. This LCDhas two registers, in particular, Commandand Data.

\subsection{INTERFACING CIRCUIT (LM358) :}

A Comparator is a gadget that thinks about two voltages or flows and yields an advanced sign demonstrating which is bigger. It has two simple information terminals and one parallel computerized yield. The yield is in a perfect world

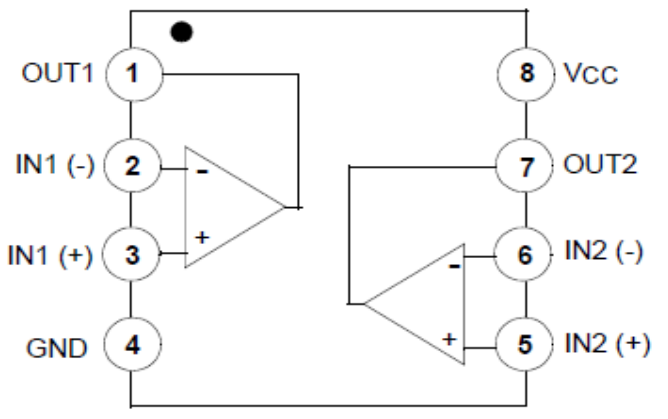

\subsection{ULTRASONIC SENSOR:}

Ultrasonic sensors are modern control gadgets that utilization sound waves over $20,000 \mathrm{~Hz}$, past the scope of human hearing, to quantify and compute separate from the sensor to a predefined target object.
Blue Eyes Intelligence Engineering \& Sciences Publication 


\subsection{PIR Sensor:}

ThisPIR (Passive Infra-Red)Sensor is apyroelectric gadget thatdistinguishes movement by estimating changes in the infrared (heat)levels discharged by encompassing articles. This movement can be identified by checking for an abrupt change in the encompassing IR designs. Atthe point when movement is identified the PIR sensoryields a high signon its yieldstick. This rationalesign can beperused by a microcontroller or used to drive atransistor to switch a higher current burden.

\section{III.INTERNET OF THINGS (IOT)}

Web of things (IoT), is another development innovation in IT division, gives internetworking to various of gadgets, for example, sensors, actuators, PLCs and other electronic implanted shrewd gadgets and controls, and different software's' and gives frameworks organize arrangement and availability, which empowers correspondence between these various gadgets for data trading.. Ongoing progressions, for example, the vision of the Internet of Things (IoT), the distributed computing model, and digital physical frameworks, offer help for the transmission and the executives of tremendous measures of information in regards to the patterns saw in ecological parameters. In this specific circumstance, the present work presents three diverse IoT-based remote sensors.

\section{IV.SOFTWARE SPECIFICATION}

ArduinoSoftware(IDE) - containsa content managerfor composing code,a message region,a content support,a toolbar withcatches for regularcapacities and aprogression of menus.It associates withtheArduinoandGenuino equipment totransfer programs andspeak with them. ComposingSKETCHES..Projects composed utilizing ArduinoSoftware(IDE) are calledoutlines. These portrayalsare written inthe word processorand are sparedwith the record augmentation. The messageterritory gives criticismwhile sparing andsending out andfurthermore shows blunders. The support showscontent yield bytheArduino Software (IDE),including total blundermessages and otherdata. The baseright-hand corner ofthe window showsthe arranged boardand sequential port.The toolbar catchesenable you tocheck and transferprograms, make, open,and spare draws, and open thesequentialscreen.NB: Versionsof the ArduinoSoftware (IDE) preceding1.0 spared outlineswith the expansion.pde. It isconceivable to openthese records withrendition 1.0, youwill be provokedto spare thesketch with the.ino augmentation onspare.

\section{DIGITAL IMAGE PROCESSING}

Computerized picturepreparing is theutilization of PCcalculations to performpicture handling onadvanced pictures. The2D ceaseless pictureis partitioned intoN lines andM sections. Theconvergence of aline and asegment is knownas a pixel.The picture canlikewise be acapacity different factorsincluding profundity, shading,and time. Apicture given as astraightforwardness, slide, photoor a Xbeamis first digitizedand put awayas a frameworkof paired digitsin PC memory.This digitized picturewould then beable to behandled as wellas showed ona high-goals TVscreen.
For showcase,the picture isput away ina quick accesscushion memory, whichinvigorates the screenat a rateof 25 outlinesfor every secondto create anoutwardly nonstop presentation.

MATLAB (frameworkresearch center) isa numerical figuringcondition and fourth-ageprogramming language.Createdby Math Works,MATLAB permits frameworkcontrols, plotting ofcapacities and information,usage of calculations,production of UIs,and interfacing withprojects written indifferent dialects, includingC, $\mathrm{C}++$, Java, and Fortran. Despitethe fact thatMATLAB is plannedprincipally for numericalfiguring, a discretionarytool compartment utilizestheMuPADsymbolicmotor,enabling access torepresentative registering abilities.An extra bundle,Simulink, includesgraphical multi-space recreationand Model-Based Designfor dynamic andimplanted frameworks. In2004, MATLAB hadaround one millionclients crosswise overindustry and thescholarly community.MATLABclients originate fromdifferent foundations ofdesigning, science, andfinancial aspects. MATLABis generally utilizedin scholastic andresearch foundations justas mechanical endeavors. MATLAB wasfirst received byscientists and professionalsin control building,Little's claim tofame, yet rapidlyspread to numerousdifferent areas. Itis presently additionallyutilized in training,specifically the educatingof straight variablebased math andnumerical investigation, andis famous amongresearchers associated withpicture handling. TheMATLAB application isworked around theMATLAB language

\section{VI.RESULT}

Advantages are simpler to appraise precisely. We have precise information on number of heart occurrences, how survival rates change when breakdown to-stun times change, and we can gauge lives spared by calculating in the episodes. The framework ought to react and the assessed improved reaction times. Nonetheless, progressively exact assessments of both expense and advantages are required before a helpful development can be performed.The main minutes after a mishap are basic and fundamental to give the correct consideration to anticipate acceleration. Accelerating crisis reaction can forestall passings and quicken recuperation drastically. This is eminently valid for heart disappointment, suffocating, injuries and respiratory issues.

\section{VII.CONCLUSION AND FUTURE SCOPE}

In this paper, we propose a product characterized observation framework (SDSS) in which a brought together controller decides the rest calendars of vitality reaping and non-energyharvesting sensor hubs. To determine the ideal rest plans limiting the quantity of dynamic sensor hubs while giving adequate observation execution, a CMDP issue is figured, and the ideal strategy for rest booking is gotten. The assessment results show that just important sensor hubs are utilized to screen focuses in the SDSS, and in this

Published By:
Blue Eyes Intelligence Engineering
\& Sciences Publication


manner the quantity of dynamic sensor hubs can be diminished by up to $90 \%$ while giving the ideal dimension of target observing likelihood. In addition, it tends to be seen that the SDSS works adaptively notwithstanding when the working condition (e.g., battery limit of a sensor hub and the between errand event rate) is changed. In our future work, we will stretch out the proposed framework to think about participatory detecting where members (e.g., cell phones or associated vehicles) screen targets and report the detected outcomes for improved observation execution. Additionally, the motivation and security instruments in the participatory detecting framework will be additionally explored.

\section{REFERENCES}

1 S. Chen, H. Xu, D. Liu, B. Hu, H. Wang, "A vision of IoT: Applications challenges and opportunities with China perspective", IEEE Internet Things J., vol. 1, no. 4, pp. 349-359, Aug. 2014.

2 M. Handte, S. Foell, S. Wagner, G. Kortuem, P. Marrón, "An Internet-of-Things enabled connected navigation system for urban bus riders", IEEE Internet Things J., vol. 3, no. 5, pp. 735-744, Oct. 2016.

3 B. Li, B. Tian, Q. Yao, K. Wang, "A vehicle license plate recognition system based on analysis of maximally stable extremal regions", Proc. 9th IEEE Int. Conf. Netw. Sens. Control, pp. 399-404, 2012.

4 C.-N. E. Anagnostopoulos, I. E. Anagnostopoulos, I. D. Psoroulas, V. Loumos, E. Kayafas, "License plate recognition from still images and video sequences: A survey", IEEE Trans. Intell. Transp. Syst., vol. 9, no. 3, pp. 377-391, Sep. 2008.

5 M. Yu, Y. D. Kim, "An approach to Korean license plate recognition based on vertical edge matching", Proc. IEEE Int. Conf. Syst. Man Cybern., vol. 4, pp. 29752980, 2000.

6 A. M. Al-Ghaili, S. Mashohor, A. R. Ramli, A. Ismail, "Vertical-edge-based car-license-plate detection method", IEEE Trans. Veh. Technol., vol. 62, no. 1, pp. 26-38, Jan. 2013.

7 J.-W. Hsieh, S.-H. Yu, Y.-S. Chen, "Morphology-based license plate detection from complex scenes", Proc. 16th IEEE Int. Conf. Pattern Recognit., pp. 176-179, 2002.

8 A. A. Lensky, K.-H. Jo, V. V. Gubarev, "Vehicle license plate detection using local fractal dimension and morphological analysis", Proc. 1st IEEE Int. Forum Strategic Technol., pp. 47-50, 2006.

9 S.-Z. Wang, H.-J. Lee, "Detection and recognition of license plate characters with different appearances", Proc. IEEE Intell. Transp. Syst., vol. 2, pp. 979-984, 2003.

10 B. Hongliang, L. Changping, "A hybrid license plate extraction method based on edge statistics and morphology", Proc. 17th IEEE Int. Conf. Pattern Recognit., vol. 2, pp. 831-834, 2004. 